\title{
Malaria cerebral en una paciente embarazada
}

\author{
Br. Carlos Mendieta Palacios, Br. Alexis Narváez Rojas. \\ Facultad de Medicina. ${ }^{1}$ \\ Recibido 1 marzo 2013-Aprobado 28 de octubre 2013.
}

\section{RESUMEN}

El manejo de la malaria es complejo por múltiples factores que inciden sobre la enfermedad siendo uno de los principales el hecho que se presenta en áreas de difícil acceso, dentro de las formas de malaria la más grave es la cerebral y no se había reportado ningún caso en los últimos diez años, por lo que se presenta el siguiente reporte de caso descriptivo. La zona de Waspán, caracterizada por ser una zona de poca accesibilidad, la malaria por $P$. falciparum es prevalente y puede terminar en un desenlace fatal. Se encontró una paciente embarazada sin historia de enfermedad cerebral pasada, ni de hipertensión arterial, no diabética quien presento 3 convulsiones tónico clónicas con 10 minutos de intervalo sin relajación de esfínter, , con presión arterial media de $106 \mathrm{mmHg}$, con examen general de orina con proteinuria negativa se descarta dicha posibilidad, con una gota gruesa con reporte de +++ para $P$. falciparum, dichas convulsiones cesaron luego de la administración de quinina, se le realiza una cesa rea por un hematoma placentario del $2 \%$, el bebé muere a las 2 horas del posquirúrgico. La malaria es una amenaza para una paciente embarazada y más aún por $P$. falciparum, por lo que en zonas endémicas debe haber una vigilancia estricta en aras de disminuir la mortalidad del embrión y el feto durante el embarazo.

Palabras clave: malaria, embarazo, Waspán, cerebral.

\section{INTRODUCCIÓN}

La Malaria es la segunda enfermedad infecciosa relacionada con la muerte más importante en el mundo después de la tuberculosis. Esta una enfermedad sistémica ocasionada por protozoarios del género Plasmodium, los cuales son transmitidos por la hembra del mosquito Anopheles spp. De las especies de Plasmodium las dos más relevantes desde el punto de vista epidemiológico son $P$. falciparum y vivax (World Health Organization 2002 y Nosten 2007). Se estima que en 2010 ocurrieron 655 mil muertes por malaria en el mundo y alrededor del $86 \%$ fueron en niños menores de 5 años, el $40 \%$ de la población mundial vive en zonas de riesgo para contraer la enfermedad, en Nicaragua se ha reportado una disminución de los casos del 50\% desde el 2000-10 (World Health Organization 2011), el total de la población en riesgo en Nicaragua para 2007 fue de 3,996,092 habitantes, de los cuales 451,197 estaban en alto riesgo (Pan American Health Organziation 2008).

La malaria gestacional ocurre aproximadamente en 50 millones de mujeres al año, aproximadamente 10 mil de ellas y 200 mil niños mueren como resultado de esta enfermedad (World Health Organization n.d.) y la Malaria Cerebral Humana $(\mathrm{MCH})$ es la complicación más severa de $P$. falciparum y ha atraído la atención de clínicos y científicos, la MCH ocurre en menos de dos semanas luego de la picadura del mosquito y se puede desarrollar luego de 2-7 días de fiebre, la definición clínica aceptada de MCH es síndrome neurológico que puede llevar al coma inexplicable por cualquier otro motivo. Para cumplir con la definición debe ser detectada la infección por P. falciparum y las otras causas de encefalitis deben ser descartadas. También se presenta convulsiones, retinopatía y alteraciones del tallo cerebral como lo explica Laurent R. et. al. (2012). Diversos autores han demostrado aumento de la susceptibilidad a

\footnotetext{
${ }^{1}$ Trabajo dirigido por los doctores Álvaro Fidel García Gómez y Clara Isabel González Moncada
} 
adquirir la enfermedad durante el embarazo. Según Nafisstou D. et. al. (2000) este padecimiento se relaciona con disminución de la respuesta Th1, típica del embarazo, que reduce la respuesta inmunitaria celular, aunada al efecto inmunodepresor debido al cortisol placentario, progesterona y otros cortico esteroides. Aumenta la citoadherencia de $P$. falciparum a la placenta, específicamente del sincitiotrofoblasto, influido por glucosaminoglucanos y disminuye la respuesta celular de los macrófagos placentario.

Aquí se presenta un caso que se confundió con preeclampsia y no se hizo diferencial con síndrome de HELLP $^{2}$ como diagnóstico diferencial.

\section{Caso clínico}

Paciente de 24 años de edad, residente de la comarca 'Santa Fe de Rio Coco' ubicada a 8 horas de Waspan, Región Autónoma del Atlántico Norte, Nicaragua. La zona que corresponde al caso comunicada es altamente endémica representando la más endémica del país, por lo que esperaríamos las manifestaciones relativamente inferiores a otras zonas del país.

Ingresó al Hospital Escuela "Bertha Calderón Roque" (HEBCR) con diagnóstico de posquirúrgica de cesárea MK, con obliteración tubárica bilateral (OTB), realizada por diagnóstico de eclampsia anteparto con desprendimiento prematuro de placenta normoinserta (DPPNI) de $25 \%$, con una presentación pélvica, recibiendo infusión de difenhidantoína y quinina en brazo derecho. Al exámen físico se encontrón tensión arterial de 100/70, frecuencia cardíaca de $90 \mathrm{lpm}$ y temperatura de $36.6^{\circ} \mathrm{C}$, al examen físico se detectó hepatomegalia y anemia clínica. El interrogatorio el cual se le realizo con apoyo de la enfermera que la acompañaba quien interpretaba el dialecto misquito no se registró antecedentes familiares crónicos de importancia pero si infeccioso como Malaria. Ingresa a la Unidad de Cuidados Intensivos, se le agrega al diagnóstico de ingreso: malaria cerebral, insuficiencia renal aguda, coagulación intravascular diseminada, hepatomegalia y anemia.

Fue reportada del puesto de salud de Santa Fe de Río Coco por un cuadro clínico de 13 días con fiebre no cuantificada, concomitaba con cefalea, mialgia, vómito y epigastralgia y tres convulsiones tónico clónicas con aproximadamente diez minutos de intervalo sin relajación de esfínteres y una gota gruesa de +++. El personal llega ocho horas después por la localización de la comunidad, la paciente es encontrada somnolienta, poco orientada, recibiendo cloroquina, quinina con clindamicina. Se le administra Hidralazina 5mg intravenosa motivada por sintomatología como cefalea y convulsiones, sin cuantificación de la tensión arterial, luego es traslada al centro de salud de Waspán donde reporta tensión de 120/90mmHg en brazo izquierdo y 120/100 mmHg en brazo derecho con presión arterial media (PAM) de $106 \mathrm{mmHg}$, con un examen general de orina que no reporta presencia de proteínas.

La paciente con 29 semanas de gestación se le realizó una ecografía que reportó un hematoma placentario de $2 \%$ por lo que se le practicó una cesárea con duración de 45 minutos encontrándose un hígado indemne, útero acorde a edad gestacional, placenta desprendida en un $25 \%$, hubo pérdida sanguínea de $500 \mathrm{ml}$, neonato femenino, con puntuación Apgar de 2/5, nace pesando mil gramos y fallece a las tres horas y treintaiocho minutos.

Al ingresar al HEBCR se le practicaron exámenes de laboratorio, que mostraron hematocrito de $25 \%$, plaquetas de $141000 \mathrm{cel} / \mathrm{mm} 3$, leucocitos de $8500 \mathrm{cel} / \mathrm{mm} 3$, enzimas hepáticas como alaninoaminotranferasa (ALT) de $24 \mathrm{UI} / \mathrm{L}$ y, aspartato-aminotransferasa (AST) de $16 \mathrm{UI} / \mathrm{L}$, química sanguínea con creatinina sérica de $0.7 \mathrm{mg} / \mathrm{dL}$, nitrógeno ureico en sangre (BUN) de $26 \mathrm{mg} / \mathrm{dL}$, ácido úrico de 7.4

\footnotetext{
${ }^{2}$ HELLP, Hemolysis, Elevated Liver enzymes y Low Platlet count.
} 
$\mathrm{mg} / \mathrm{dL}$, bilirrubina total $1.9 \mathrm{mg} / \mathrm{dL}$ y bilirrubina indirecta de $1 \mathrm{mg} / \mathrm{dL}$. El examen general de orina (EGO) no demostró proteínas. Con los datos clínicos y paraclíncos se estableció el diagnóstico de Malaria cerebral en base al reporte de $P$. falciparum en la gota gruesa y con manifestaciones del sensorio, sin criterio de preclampsia o síndrome de HELLP. Se ingresa a UCI con orientaciones de respetar nada por oral (NPO), sonda nasográstrica (SNG), solución mixta $300 \mathrm{ml}$ cada ocho horas y se agrega dextrosa de $500 \mathrm{ml}$ con quinina de $1200 \mathrm{ml}$ intravenosa (IV) pasada en cuatro horas y se establece metamizol un $1 \mathrm{gr}$ IV. Se le realiza visita conjunta con médico de base de medicina interna donde es encontrada anúrica e ictérica, con tiempo de protrombina (TP) de 15' y tiempo parcial de tromboplastina (TPT) de 37', una gota gruesa que reportó $P$. falciparum + , sin reporte parasitario en frotis con hipocromía del $40 \%$, normocromía del $60 \%$ y microcitosis del $30 \%$, se considera la paciente en falla renal.

A los dos días de ingreso se reporta sin datos de encefalopatía hipertensiva y con un hematócrito posttransfusión de $28 \%$, se registra una presión arterial de $120 / 100 \mathrm{mmHg}$ por lo que se decide administra un bolo de labetalol, estas presiones siguen en aumento hasta 150/100 $\mathrm{mmHg}$ (con PAM de 123.3mmHg) agregándosele otro bolo de labetalol de $20 \mathrm{mg}$ stat y nifedipina de $20 \mathrm{mg}$ por vía oral (PO) cada ocho horas, con poco éxito el mismo día se presenta con 140/100mmHg y se modifica a atenolol 100mg PO cada doce horas y nifedipino PO cada 6 horas, al evaluársele el calcio se determina $7.63 \mathrm{mEq} / \mathrm{L}$ (corregido a $8.19 \mathrm{mg} / \mathrm{dL}$ ) y potasio de $6.83 \mathrm{mEq} / \mathrm{L}$, por lo que se le administra gluconato de calcio a un gramo IV cada doce horas, hipertensión continua y se le agrega captopril de $50 \mathrm{mg}$ cada ocho horas y alfametildopa a 500mg PO cada seis horas, hasta ese momento habían pasado 72 horas desde el último evento convulsivo y se omitió Difenihidantoina. Se logra la mejoría clínica y disminución de la tensión arterial hasta $110 / 70 \mathrm{mmHg}$ y se agrega hidroclorotiazida $25 \mathrm{mg}$ PO cada doce horas, la paciente mejora su condición y es dada de alta de la Unidad de Cuidados Intensivos a los 6 días de estancia. Los exámenes de laboratorio se muestran en el cuadro 1.

\section{DISCUSIÓN}

Cuadro 1. Datos de laboratorio

\begin{tabular}{lll}
\hline Prueba de laboratorio & Ingreso & $\begin{array}{l}\text { Egreso } \\
\text { de UCI }\end{array}$ \\
\hline Hematócrito (\%) & 25 & 37 \\
Plaquetas (cel/mm ${ }^{3}$ ) & 141000 & 313000 \\
Ácido úrico(mg/dL) & 7.4 & 6.9 \\
Bilirrubina ind. & 1 & 0.2 \\
(mg/dL) & 24 & 41 \\
ALT (UI) & 16 & 39 \\
AST (UI) & 5.08 & 4.5 \\
Proteínas en orina &
\end{tabular}

Según Menéndez (1995) malaria es una causa frecuente de morbilidad y mortalidad materna en zonas endémicas, con mayor número de casos en el África subsahariana. Por ejemplo Piñeros (2002) y Diagne et. al. (2000) mencionan que los efectos en el embarazo, feto y neonato dependen del grado de inmunidad adquirida por la madre antes del embarazo, y la eficacia de su respuesta inmunológica durante, el embarazo, así como de la intensidad de la transmisión de la enfermedad (eventualmente esto dependerá de la zona endémica, aquellas zonas que sean endémicas tendrán una población con mejor inmunidad antipalúdica).

Tanto Diagne et. al. (2000) como Dellicour et. al. (2007) demostraron que las madres pueden tener la infección durante la gestación y cursar subclínicamente pero con anemia y parasitemia placentaria. Entre los efectos de la malaria gestacional encontramos: disminución del crecimiento intrauterino y bajo peso al nacer en zonas endémicas, sin embargo en aquellas áreas que no lo son la enfermedad progresa de manera más grave hasta anemia grave en el $15 \%$ de los casos, aborto, parto prematuro en un $36 \%$ con restricción de crecimiento hasta en un 70\%, con muerte neonatal del 3-8\%. Según Taylor (1990 y 1993) los síntomas neurológicos son frecuentemente asociados a acidosis metabólica severa, anemia e hipoglicemia. Sin embargo, acorde a Haldar et. al. (2007) la exclusión de todos estos factores son importantes para la definición de la verdadera Malaria Cerebral Humana. No existe un consenso sobre la patogénesis de la HCM, en realidad, este tema ha sido uno de los más dogmáticos y divisorios en la investigación sobre 
malaria. Esto se debe al hecho que los estudios en humanos son muy limitados, muchos estudios desde 1900 como los de Durk (1917), Gaskel (1920) y varios autores más han reportado oclusiones capilares en el cerebro, edema endotelial y un secuestro de células rojas infectadas (IRBC ${ }^{3}$ ). En los últimos 20 años, estudios como el de Dorovini (2011) con muestras mayores han llevado a una definición de MCH mucho más rigurosa dándole soporte a los estudios anteriores. Estos resultados han llevado al dogma prevalente que el secuestro cerebral de IRBC es el mecanismo etiológico que lleva a la HCM.

El mecanismo por el cual el secuestro lleva a complicaciones neurológicas y muerte aún no está definida claramente. Henri C. van der Heyde postuló una hipótesis unificada para la génesis de la malaria cerebral que propone que los antígenos parasitarios activan plaquetas que contribuyen a la activación de la respuesta inflamatoria e incrementa los niveles de moléculas endoteliales de adhesión celular (eCAMs). Los altos niveles de eCAMs resultan en un secuestro parásito-eritrocito e inflamación local marcada que puede dañar la microvasculatura cerebral que no puede ser reparado por el sistema de hemostasis por el estado procoagulantivo, esto da origen a un filtrado o hemorragia cerebral, procesos similares puede ocurrir en otros órganos como el pulmón. En la infección por Plasmodium la anemia es un proceso debido a la hemólisis inicial ocasionada por la liberación de los merozoitos, y que se intensifica por alteraciones en la eritropoyesis por la infección de la médula ósea. Una vez establecida la infección hay alteraciones de los fosfolípidos de membrana del eritrocito que modifican la maleabilidad eritrocitaria y aumentan su destrucción, aquellos eritrocitos que están infectados se encuentran secuestrados en la microcirculación, invaden la circulación esplénica, y de esta forma aumentan su vida media intravascular. La interacción dentro del eritrocito genera moléculas de adhesión celular que interactúan con ligandos endoteliales (trombospondina y el receptor CD 36), lo que ocasiona su agregación y disminuye el flujo sanguíneo y la hipoxia tisular. La reacción inflamatoria induce la liberación del factor de necrosis tisular (FNT- $\alpha$ ) que, una vez liberado, genera daño endotelial difuso, aumenta la permeabilidad vascular y, por consecuencia, produce edema intersticial secundario.

Ochoa (2004), Malagón (2005), Mandell \& Dolin, (2005), (Brito, 2007) y Monckenhaupt, (2006) soportan lo anterior y coinciden en que los cambios en el endotelio vascular también incluyen la expresión del factor tisular, la activación del sistema de coagulación y la evolución de la coagulación intravascular diseminada, que disminuye y perpetúa la isquemia tisular sistémica; además, aumentan las moléculas de adhesión celular; y hay mayor secuestro de eritrocitos infectados. De esta manera, según Rogerson (2007) la infección de los eritrocitos en la madre y la reacción inflamatoria secundaria a la activación endotelial hacen que la infección por Plasmodium comparta signos y síntomas con la preeclampsia y el síndrome HELLP. Algunos estudios encontraron relación entre el paludismo y el riesgo de preeclampsia e hipertensión en el embarazo. El paludismo gestacional puede afectar la circulación placentaria, si la infección se manifiesta durante el periodo de invasión trofoblástica, en especial durante elperiodo de reestructuración de las arterias espirales, como ocurre en la preeclampsia.

Este mismo estudio informa que el riesgo de hipertensión aumento en primeros embarazos con variantes de malaria placentaria, inflamatoria y crónica; esta respuesta podría deberse a una respuesta fetal a la inflamación placentaria. Aunque la paciente que se está comunicando, no tenía fiebre al ingreso, el examen de gota gruesa permitió identificar al parásito durante los paroxismos febriles en su estancia en la sala de cuidados intensivos.

\footnotetext{
${ }^{3}$ IRBC, Infected Red Blood cells
}

\begin{tabular}{|c|c|c|c|c|}
\hline $\begin{array}{l}\text { Datos Clínicos y } \\
\text { de laboratorio del } \\
\text { síndrome de } \\
\text { HELLP }\end{array}$ & Criterios de Sibai & $\begin{array}{c}\text { Criterios de } \\
\text { Martin }\end{array}$ & $\begin{array}{l}\text { Frecuencia en el } \\
\text { síndrome de } \\
\text { HELLP }\end{array}$ & $\begin{array}{c}\text { Frecuencia en } \\
\text { la Malaria }\end{array}$ \\
\hline \multicolumn{5}{|l|}{ Hemólisis } \\
\hline Bilirrubina total & $1.2 \mathrm{mg} / \mathrm{dL}$ & $1.6 \mathrm{mg} / \mathrm{dL}$ & $100 \%$ & $>30 \%$ \\
\hline $\begin{array}{l}\text { Deshidrogenasa } \\
\text { láctica }\end{array}$ & $600 \mathrm{UI}$ & $1200 \mathrm{UI}$ & $100 \%$ & $80 \%$ \\
\hline $\begin{array}{l}\text { Elevación de } \\
\text { enzimas } \\
\text { hepáticas }\end{array}$ & $\mathrm{TGO}>70$ & $\begin{array}{l}T G O>48 \\
T G P>24\end{array}$ & $100 \%$ & $25 \%$ \\
\hline Plaquetopenia & $\begin{array}{l}<100000 \\
\mathrm{cel} / \mathrm{mm}^{3}\end{array}$ & $\begin{array}{l}<100000 \\
\mathrm{cel} / \mathrm{mm}^{3}\end{array}$ & $>80 \%$ & $70 \%$ \\
\hline Dolor hepático & & & $63 \%$ & $60 \%$ \\
\hline Nausea y vómitos & & & $36 \%$ & $80 \%$ \\
\hline Cefalea & & & $33 \%$ & $>90 \%$ \\
\hline Hipertensión & & & $85 \%$ & No hay \\
\hline Proteinuria & & & $96 \%$ & No hay \\
\hline
\end{tabular}


Mandel \& Dollin (2005) recogen los hallazgos paraclínicos más frecuentes: trombocitopenia en un 70\% de los casos $(<150000 \mathrm{cel} / \mathrm{mL})$, anemia en más del 25\%, leucocitos en menos del $5 \%$ y elevación de las enzimas hepáticas en $25 \%$, la hiperbilirrubinemia se identifica en el $30 \%$ de los casos y la elevación de la deshidrogenasa láctica en más del $80 \%$. Los criterios para síndrome de HELLP están detallados en el cuadro 2.

\section{CONCLUSIÓN}

La pacientes embarazas tienen mucha susceptibilidad y riesgo de Este caso ilustra como las zonas endémicas deben ser consideradas para diagnóstico diferencial de síndrome de HELLP.

\section{BIBLIOGRAFÍA}

Brito BB, Castro GC, Vidal GV (2007) Medicina crítica y terapia intesiva: Síndrome de HELLP y otras microangiopatías, ( $1^{\mathrm{a}}$ ed.). México: Intersistema editores

Dellicour, S. Hall, S. et al. (2007). 'The safety of artesamisins during pregnancy: a pressing question', Malaria Journal, vol 6, pp. 6-15.

Diagne, N. Rogier, C. et al. (2000). 'Increased susceptibility to malaria during early postpartum period' $N$ Eng J Med, vol 343, no 9, pp. 598-603.

Dorovini-Zis, Schmidt K, Huynh H, Fu W, et al (2011). 'The neuropathology of fatal cerebral malaria in Malawian children, Am J Pathol, vol 178, issue 5, pp. 2146-2158.

Durk H. (1917). 'Uber die bei malaria comatose aufretenden veranderungen des zentranervensystem' Archiv Schiffs Tropenhygien, vol 2, no 21, pp. 317-322.

Gaskel SJ, Millar W. L. (1920). 'Studies on Malignant Malaria in Macedonia' QJM: An international Journal of Medicine, vol 13, issue 52, pp. 381-426.

Laurent, R. et. al. (2012). 'Cerebral malaria, Mysteries at the blood brain barrier' Virulenc, vol 3, issue 2, pp. 1.

Malagón F. (2005) 'El origen del paroxismo malárico' Rev Med IMSS, vol 43, no 1, pp. 83-88.

Mandell Benet \& Dolin (2005) Principles and practice of infectious disease, (6th ed.). Philadelphia: Elsevier.

Menéndez, C. (1995) 'Malaria during pregnancy: a priority area of malaria research and control', Elsevier, vol 11, issue 5, pp 178-183.

Mochenhaupt FP, Bedu-Addo G et al (2006) 'Detection and clinical manifestation of placental malaria in southern Ghana', Malar J, vol 5, pp. 1-10.

Nafisstou, D. et al. (2000). 'Increased susceptibility to Malaria during the Early Postpartum period' $N$ Engl J Med, vol 343, no 9, pp 600-603.

Nosten F, McGready R, Mutabingwa T. (2000). 'Case management of malaria in Pregnancy', Lancet Infect Dis, vol 7, issue 2, pp. 25.

Pan American Health Organization, Regional office of the World Health Organization. (2008). Malaria in Nicaragua 2000-07, Time series Epidemiological Data from 2000 to 2007, Pan American Health Organization, Regional office of the World Health Organization.

Piñeros, G. y Blair, S. (2002). 'Malaria y embarazo', Infectio, vol 6, no 3, pp. 168-176.

Rogerson, SJ. Hvild L, Duffy PE. et. al. (2007). 'Malaria in pregnancy: Pathogenesis and immunity', Lancet Infect Dis, vol 7, issue 2, pp. 105-117.

Taylor TE, Wirima JJ, Molyleum ME et al. (1990). 'Hypoglycaemia and cerebral malaria', Lancet, vol 336, issue 8720, pp. 950-951.

World Health Organization. (2002). Roll back malaria, a global partnership, visto el 12 de septiembre de 2012 en la URL: http://www.rbm.who.int/. 